\title{
SECOND ORDER TWO-PARAMETRIC QUANTUM BOUNDARY VALUE PROBLEMS
}

\author{
YOUSEF GHOLAMI
}

Abstract. In this paper we study second order two-parametric quantum boundary value problems. The main aims of this paper are presented in two steps. In the first step, we consider second order two-parametric quantum boundary value problems with general nonlinearities and by the use of Krasnoselskii fixed point theorem on positive cones we provide some sufficient conditions to reach the existence, multiplicity and nonexistence of positive solutions. At the and of this step, some illustrative examples are given to show practical implementability of the obtained theoretical results. In the second step, we consider the corresponding two-parametric quantum eigenvalue problems and in the light of Lyapunov inequalities, we present a lower bound estimation for positive eigenvalues. We complete this step with a numerical evaluation to identify validity of the obtained lower bound.

Mathematics subject classification (2010): 05A30, 39A13, 34B05, 34B18, 34B27, 47H10, $26 \mathrm{D} 15$.

Keywords and phrases: Two-parametric quantum operators, Dirichlet-Neumann boundary value problems, Green function, fixed point theory, positive solutions, nonexistence, Lyapunov inequality, lower bound estimation for positive eigenvalues.

\section{REFERENCES}

[1] W. A. Al-S Alam, q-analogues of Cauchy's formulas, Proc. Amer. Math. Soc. Vol. 17, No. 4, (1996), pp. 616-621.

[2] G. A. Anastassiou, Multivariate Lyapunov inequalities, Appl. Math. Lett. Vol. 24, (2011), pp. 2167-2171.

M. H. Annaby, Z. S. Mansour, q-fractional calculus and equations, Springer, (2012).

[3] M. H. ANNABy, Z. S. Mansour, $q$-Taylor and interpolation series for Jackson $q$-difference operators, J. Math. Anal. Appl. Vol. 344, (2008), pp. 472-483.

[4] A. CABADA, J. B. FERREIRO, Existence of positive solutions for nth-order periodic difference equations, J. Difference Equ. Appl. Vol. 17, No. 6, (2011), pp. 935-954.

[5] S. S. ChEnG, A discrete analogue of the inequality of Lyapunov, Hokkaido Math. J. Vol. 12, (1983), pp. 105-112.

[6] S. DHAR, Q. Kong, Liapunov-type inequalities for third-order half-linear equations and applications to boundary value problems, Nonlinear Anal. Theory, Methods and Applications. 110 (2014), pp. 170181.

[7] M. El-Shahed, H. A. Hassan, Positive solutions of q-difference equation, Proc. Amer. Math. Soc. Vol. 138, No. 5, (2010), pp. 1733-1738.

[8] L. H. ERBE, H. WANG, On the existence of positive solutions for ordinary differential equations, Proc. Amer. Math. Soc. Vol. 120, No. 3, (1994), pp. 743-748.

R. A. C. FERREIRA, A Lyapunov-type inequality for a fractional boundary value problem, Fract. Calc. Appl. Anal, Vol. 16, No. 4, (2013), pp. 978-984; DOI: 10.2478/s13540-013-0060-5.

[9] G. Gasper, M. Rahmen, Basic Hypergeometric Series, Cambridge University Press, Cambridge, (2004).

[10] Y. GHOLAmi, Lyapunov inequalities for two-parametric quantum Hamiltonian systems and their applications, Differ. Equ. Appl. Vol. 10, No. 3, (2018), pp. 261-276. 
[11] R. W. Gosper, JR., S. K. Suslov, Numerical investigation of basic Fourier series, Contemporary Mathematics, Vol.254, (1999).

[12] V. Gupta, TH. M. Rassias, $(p, q)$-Genuine Baskakov-Durrmeyer operators, Int. J. Nonlinear Anal. Appl. Vol. 7, No. 2, (2016), pp. 69-76.

G. S. Guseinov, B. KaymaKÇAlan, Lyapunov inequalities for discrete linear Hamiltonian systems, Comput. Math. Appl, Vol. 45, (2003), pp. 1399-1416.

[13] P. Hartman, Ordinary Differential Equations, John Wiley \& Sons, New York, (1964).

[14] J. HENDERSON, R. LUCA, Existence of positive solutions for a system of second-order multi-point discrete boundary value problems, J. Difference Equ. Appl. Vol. 19, No. 11, (2013), pp. 1889-1906.

[15] J. Henderson, R. LUCA, On a second-order nonlinear discrete multi-point eigenvalue problem, J. Difference Equ. Appl. Vol. 20, No. 7, (2014), pp. 1005-1018.

[16] J. Henderson, R. LuCA, On a multi-point discrete boundary value problem, J. Difference Equ. Appl. Vol. 19, No. 4, (2013), pp. 690-699.

[17] F. H. JACKSON, On q-Functions and a certain difference operator, Transactions of the Royal Society of Edinburgh, Vol. 46, No. 2, (1909), pp. 253-281.

[18] F. H. JACKSON, q-Difference equations, Am. J. Math. Vol. 32, (1910), pp. 305-314.

[19] R. JAGANNATHAN, K. SRINIVAS A RAO, Two-parameter quantum algebras, twin-basic numbers, and associated generalized hypergeometric series, arXiv:math/0602613v1, (2006).

[20] V. Kac, P. Cheung, Quantum calculus, Springer Science \& Business Media, (2001).

[21] M. A. Krasnosels KII, Positive Solutions of Operator Equations, Noordhoff, Groningen, (1964).

[22] R. LUCA, Existence of positive solutions for a second-order $m+1$-point discrete boundary value problem, J. Difference Equ. Appl. Vol. 18, No. 5, (2012), pp. 865-877.

[23] A. M. Lyapunov, The general problem of the stability of motion, Int. J. Control, Vol. 55, No. 3, (1992), pp. 521-790. http://www. tandf online.com/toc/tcon20/55/3.

[24] R. MA, Y. N. RAFFOUL, Positive solutions of three-point nonlinear discrete second order boundary value problem, J. Difference Equ. Appl. Vol. 10, No. 2, (2004), pp. 129-138.

[25] C. A. Nelson, M. G. Gartley, On the zeros of the q-analogue exponential function, J. Phys. A. Vol. 27, (1994), pp. 3857-3881.

[26] P. NJiONOU SADJANG, On the fundamental theorem of $(p, q)$-calculus and some $(p, q)$-Taylor formulas, arXiv:1309.3934v1, (2013).

[27] B. G. PAChPATte, Lyapunov tye integral inequalities for certain differential equations, Georgian Math. J, Vol. 4, No. 2, (1997), pp. 139-148.

P. M. Rajkovic, S. D. Marinkovic, M. S. Stankovic, Fractional integrals and derivatives in q-calculus, Appl. Anal. Discrete Math. Vol. 1, (2007), pp. 311-323.

[28] J. P. SUn, Y. H. ZHAO, Multiplicity of positive solutions of a class of nonlinear fractional differential equations, Comput. Math. Appl. Vol. 49, (2005), pp. 73-80.

[29] S. K. Suslov, An introduction to basic Fourier series, Springer, (2003).

[30] J. TARIBOON, S. K. NTOUYAS, Quantum integral inequalities on finite intervals, J. Inequal. Appl. Vol. 2014, No. 1, (2014), p. 121.

[31] J. TARIBOON, S. K. NTOUYAS, Quantum calculus on finite intervals and applications to impulsive difference equations, Adv. Difference Equ. Vol. 2013, No. 1, (2013), p. 282.

[32] P. J. Y. Wong, Positive solutions of difference equations with two-point right focal boundary conditions, J. Math. Anal. Appl. Vol. 224, (1998), pp. 34-58. 